\title{
Neuraminidase Inhibition Assay
}

National Cancer Institute

\section{Source}

National Cancer Institute. Neuraminidase Inhibition Assay. NCI Thesaurus. Code

C120696.

A type of enzyme-based assay that assesses neuraminidase activity in the presence of an enzyme inhibitor. 